\title{
Holographic Lattices Give the Graviton an Effective Mass
}

\author{
Mike Blake, ${ }^{1}$ David Tong, ${ }^{1}$ and David Vegh ${ }^{2}$ \\ ${ }^{1}$ Department of Applied Mathematics and Theoretical Physics, University of Cambridge, Cambridge CB3 OWA, United Kingdom \\ ${ }^{2}$ Theory Group, Physics Department, CERN, CH-1211 Geneva 23, Switzerland
}

(Received 8 November 2013; published 20 February 2014)

\begin{abstract}
We discuss the dc conductivity of holographic theories with translational invariance broken by a background lattice. We show that the presence of the lattice induces an effective mass for the graviton via a gravitational version of the Higgs mechanism. This allows us to obtain, at leading order in the lattice strength, an analytic expression for the dc conductivity in terms of the size of the lattice at the horizon. In locally critical theories this leads to a power law resistivity that is in agreement with an earlier field theory analysis of Hartnoll and Hofman.
\end{abstract}

DOI: 10.1103/PhysRevLett.112.071602

Introduction.-Here's a simple question. Take a quantum field theory at nonzero temperature and finite background charge density. What is the resistance of such a system to a dc current?

If the field theory has translational invariance, this simple question has a simple answer: the resistance is zero and the material is a perfect conductor. This is true for trivial reasons. Translational invariance implies momentum conservation which, in turn, means that there is no mechanism to dissipate the current. To extract something more interesting, we have to work a little harder and introduce effects that break the translational symmetry such as impurities or a background lattice.

Progress can be made if the breaking of translational invariance does not change the infrared fixed point of the theory. This means that, from the IR perspective, the effects can be captured by the addition of irrelevant operators $\mathcal{O}$ to the Hamiltonian, $H=H_{0}+\epsilon \mathcal{O}\left(k_{L}\right)$, where $k_{L}$ is the characteristic momentum of the underlying lattice or impurity. It was shown in $[1,2]$ that such an interaction gives rise to momentum relaxation rate, $\Gamma$, and hence resistivity, given by the retarded Green's function,

$$
\Gamma \sim \epsilon^{2} k_{L}^{2} \lim _{\omega \rightarrow 0} \frac{\operatorname{Im} G_{\mathcal{O O}}^{R}\left(\omega, k_{L}\right)}{\omega} .
$$

This is an interesting formula. Because it involves the spectral density of the operator $\mathcal{O}$ at momentum $k_{L}$, if there is to be any significant momentum dissipation-say, enough to give the resistivity $\rho$ a power-law dependence on temperature $T$-then there must be low-energy $\omega \rightarrow 0$ excitations at momentum $k_{L}$. If not, the relaxation rate will be Boltzmann suppressed.

Fermi surfaces provide a natural context in which one has light degrees of freedom at finite momentum. Such modes are simply electrons scattering across the Fermi surface with a net momentum transfer. Applying (1), with the operator $\mathcal{O}$ taken to be the four-fermion Umklapp
PACS numbers: 11.25.Tq, 04.50.Kd, 05.60.Gg, 72.10.-d

operator, reproduces the well known $\rho \sim T^{2}$ behavior of the resistivity of Fermi liquid theory.

There is another, more exotic, way to get low-energy modes at finite momentum. At critical points, excitations have a typical dispersion relations $\omega \sim k^{z}$, with $z$ the dynamical exponent. In the limit $z \rightarrow \infty$, this dispersion relation broadens out. Such theories are known as locally critical and arise naturally in the framework of holography in the guise of infrared $\mathrm{AdS}_{2}$ regions of spacetime. In such theories, time scales but space does not and the dimension of an operator $\mathcal{O}\left(k_{L}\right)$ is dependent on the momentum $k_{L}$. In [2], Hartnoll and Hofman showed that, when applied to such local critical theories, the formula (1) gives a powerlaw resistivity,

$$
\rho \sim T^{2 \Delta_{k_{L}}}
$$

where the exponent, $\Delta_{k_{L}}$ is the frequency space scaling dimension of the operator and depends on the lattice spacing $k_{L}$.

The arguments of [2] sketched above are purely field theoretic. Given that locally critical theories arise naturally in holography, one can also try to derive the scaling (2) using holographic methods alone. The appropriate holographic lattices were introduced in [3] where Einstein's equations were solved numerically (see also [4-6] for related work). Here strong evidence was presented that the dc conductivity indeed obeys (2) with $\mathcal{O}$ given by the charge density. However, this evidence relied heavily on numerics. The purpose of the present Letter is, in part, to gain an analytic understanding of this scaling behavior in a purely holographic framework. Before describing this, there is another thread that we would like to weave into the discussion.

A different approach to incorporating momentum dissipation in holographic models was introduced in [7]. The basic idea is straightforward: momentum conservation in the boundary theory follows from diffeomorphism 
invariance in the bulk. If you want to model a theory without momentum conservation, you need to consider a bulk theory without diffeomorphism invariance. Such theories usually go by the name of massive gravity.

The closet of massive gravity contains both skeletons and ghosts. There has been recent progress in constructing a (seemingly) consistent theory of a propagating massive spin 2 particle [8]. However, in the context of holographic massive gravity, life is likely to be somewhat easier. To capture momentum dissipation, you only need to give a mass to the gravitons with polarisation parallel to the boundary. This means that the bulk theory retains diffeomorphism invariance in both time and radial directions. In particular, since the timelike components of the graviton do not get a mass, it seems likely that the constraints imposed by ghosts are much weaker, if not completely absent.

The appeal of massive gravity is that, in contrast to explicit lattices or impurities, it is analytically tractable. Moreover, various aspects of thermodynamics and transport in holographic massive gravity have been explored and give encouragingly sensible answers. The low-frequency optical conductivity exhibits a Drude peak [7,9], with the momentum relaxation rate of the boundary theory determined by the graviton mass $[9,10]$. In particular, a universal formula for the dc conductivity was presented in [10]. This formula, which holds at finite temperature and chemical potential, relates the resistivity of the boundary field theory to the mass of the graviton evaluated on the horizon of the bulk black hole.

Massive gravity provides a phenomenological way to implement momentum dissipation in holography. But its microscopic origins remain mysterious and it is unclear how one can derive it from better motivated models. A second goal of this Letter is to shed some light on this.

The purpose of this letter is to draw these threads together. We start by considering Einstein-Maxwell theory in $\mathrm{AdS}_{4}$, coupled to a neutral scalar field. Translational invariance is broken by introducing a spatially modulated source for the scalar; this is precisely the setup studied in [3]. However, rather than solving the bulk equations numerically, we instead work perturbatively in the strength of the background lattice. We will see that, to leading order, the bulk conductivity calculation simplifies tremendously, with only a handful of fields responding to an applied electric field on the boundary.

Foremost among the bulk modes is a phonon-a Goldstone boson arising from the lattice. Because of bulk diffeomorphism invariance, this phonon is eaten by the metric to give an extra propagating graviton degree of freedom. The net result is a Higgs mechanism for gravity, with the graviton gaining a radially-dependent effective mass, determined by the profile of the bulk lattice. We will show that the equations describing the perturbations of the holographic lattice coincide with those arising from massive gravity. This allows us to import the result of [10], relating the resistivity to the mass of the graviton at the black hole horizon. Our punch line is that this formula reproduces the expected temperature dependence that arises from (2) in locally critical theories.

The holographic lattice.-We work with the familiar Einstein-Maxwell theory in $d=3+1$ dimensions with negative cosmological constant. To this we add to a neutral scalar field, $\phi$, of mass $m^{2} \leq 0$ so that this field corresponds to a relevant or marginal operator, $\mathcal{O}$, in the boundary theory.

The workhorse solution for applications of holography is the Reissner-Nordström black hole, describing the boundary field theory at temperature $T$ and chemical potential $\mu$. This will be our starting point. When $T \ll \mu$, it is well known that the solution asymptotes to an $\mathrm{AdS}_{2} \times \mathbf{R}^{2}$ geometry in the infrared. This reflects the fact that the boundary theory flows to a locally critical fixed point.

We now break translational invariance by introducing a spatially modulated source for the operator $\mathcal{O}$. For static solutions the scalar field can be expanded near the boundary as

$\phi_{0}(r, x, y) \sim \phi_{-}(x, y)\left(\frac{r}{L}\right)^{\Delta_{-}}+\phi_{+}(x, y)\left(\frac{r}{L}\right)^{\Delta_{+}}+\cdots$,

where $\Delta_{ \pm}=3 / 2 \pm \sqrt{9 / 4+m^{2} L^{2}}$. For technical simplicity, we will work with the standard quantisation which means that we impose a source by fixing the leading falloff, $\phi_{-}$. Here we choose to work with the striped source $\phi_{-}=$ $\epsilon \cos \left(k_{L} x\right)$ where $\epsilon$ is a small number that allows us to treat the lattice perturbatively. Turning on this source is equivalent to turning on a spatially modulated potential in the boundary theory, somewhat analogous to the optical lattices in cold atom experiments. As usual the subleading falloff, $\phi_{+}$, has the interpretation of the expectation value of the dual operator $\mathcal{O}$ in the boundary theory.

The radial profile of the lattice is dynamically determined by the scalar wave equation in the bulk. At leading order in $\epsilon$, we can work with the Reissner-Nordström geometry. The bulk solution takes the form $\phi(r, x, y)=\epsilon \phi_{0}(r) \cos \left(k_{L} x\right)$, where the background lattice profile $\phi_{0}(r)$ satisfies

$$
\frac{d}{d r}\left(\frac{f}{r^{2}} \frac{d \phi_{0}}{d r}\right)-\frac{k_{L}^{2}}{r^{2}} \phi_{0}-\frac{m^{2} L^{2}}{r^{4}} \phi_{0}=0,
$$

with $f(r)$ the familiar emblackening factor of the ReissnerNordström metric. A typical solution to this equation has $\phi$ bounded everywhere in the bulk, with its size controlled by $\epsilon$. This makes a perturbative treatment possible. Our goal in this Letter is to calculate the resistivity due to the lattice to order $\mathcal{O}\left(\epsilon^{2}\right)$. By turning on a lattice in the scalar field, as opposed to the chemical potential, we have ensured that the stress tensor of our lattice is smaller than the lattice itself, that is $\mathcal{O}\left(\epsilon^{2}\right)$. This means that, to leading order, we can 
ignore any oscillations in the metric and gauge field. Furthermore, it was shown in [10] that, in the context of massive gravity, the dc conductivity is independent of the homogeneous corrections to the background. We will see shortly that the same result holds here too.

The net result of these simplifications is that, in order to compute the dc conductivity, we may treat the background geometry as being the Reissner-Nordström black hole, with an oscillating scalar lattice sitting on top.

We now perturb the lattice background to determine the conductivity. We do this by adding a small electric field on the boundary of the form $\delta A_{x} e^{-i \omega t}$. We impose ingoing boundary conditions at the IR horizon to determine the solution $\delta A_{x}(r ; \omega)$, the optical conductivity is then given by $\sigma(\omega)=\delta A_{x}^{\prime} /\left.\left(i \omega \delta A_{x}\right)\right|_{r=0}$.

In the usual case of a homogeneous black hole, $\delta A_{x}$ sources a metric perturbation $\delta g_{t x}$ but, if we work in gauge $\delta g_{r x}=0$, nothing more. In contrast, in the full lattice background, studied numerically in [3], things are much more complicated and one ends up having to solve for 11 coupled perturbations. Thankfully, in our small-lattice expansion, things are not so bad. We can continue to work in the gauge $\delta g_{r x}=0$. We have already argued that to leading order it is consistent to treat the background metric and gauge field as homogeneous. As a result, the metric perturbation $\delta g_{t x}$ sources an inhomogeneous scalar perturbation $\delta \phi$, but there things stop.

The upshot is that we have three perturbations: $\delta A_{x}, \delta g_{t x}$ and $\delta \phi$, together with the constraint equation that arises from the gauge fixing condition $\delta g_{r x}=0$. Before we jump into a morass of coupled equations, let us first explain some of the physics that underlies these perturbations.

We start with the new ingredient which is the scalar perturbation $\delta \phi$. A simple parity argument ensures that the scalar perturbation takes the form, $\delta \phi(r, x, t)=$ $\delta \phi(r, t) \sin \left(k_{L} x\right)$. However, there is deeper interpretation of this functional form: it is a bulk phonon mode. This is easily seen by rewriting the perturbation as a position dependent phase of the bulk lattice,

$$
\phi(r, x, t)=\epsilon \phi_{0}(r) \cos \left(k_{L}[x-\pi(r, t)]\right) .
$$

The phonon mode $\pi$ is related to the scalar perturbation by $\delta \phi=\epsilon k_{L} \phi_{0}(r) \pi(r, t)$. At each radial slice in the bulk, you can think of a layer of material with "ions" (i.e., peaks of the lattice) positioned at $x-\pi(r, t)=2 \pi n / k_{L}$. A nonzero momentum in the bulk, $\delta g_{t x}$, collides with these layers and shifts them relative to one another. This disturbance then propagates as a transverse phonon in the radial direction until it reaches the horizon where the momentum is lost to the system. This simple picture makes it clear that the phonon is responsible for the momentum dissipation in the boundary theory and that this dissipation is ultimately governed by the properties of the horizon. This will be manifest in our formula below for the dc conductivity.
The existence of this bulk phonon mode is intimately tied with the fact that the lattice induces a mass for the graviton. To see this, we can use diffeomorphism invariance to freeze the phonon mode at the expense of introducing a new, propagating degree of freedom in the metric. All we need to is to switch to a new coordinate defined by $\tilde{x}=x-\pi(r, t)$. This coordinate transformation places the dynamics back into the metric. In this new gauge, $\delta g_{r x}$ becomes dynamical and corresponds to the extra polarisation of a massive graviton. This is entirely analogous to the Higgs mechanism in gauge theory where a would-be Goldstone mode is eaten by the gauge field. Here, instead, the phonon is eaten by the metric. The whole discussion parallels the usual Stückelberg formulation of massive gravity [11], now with the phonon playing the role of the Stuickelberg field. (See also [12]).

To truly see that our lattice describes a massive graviton, we should look at the full perturbation equations below. But there is a quick, cheap way to get the basic idea. From the discussion above, it is clear that the mass should arise from the breaking of translational invariance. In other words, it comes from the $\left(\partial_{x} \phi\right)^{2}$ terms in the action. Evaluated on the background solution $\phi=\epsilon \phi_{0}(r) \cos \left(k_{L} x\right)$, the homogeneous contribution to the mass is

$$
S_{\text {eff }}=\frac{1}{2} \int d^{4} x \sqrt{-g} M^{2}(r) g^{x x},
$$

where the effective mass $M(r)$ is radially dependent and given by

$$
M^{2}(r)=\frac{1}{2} \epsilon^{2} k_{L}^{2} \phi_{0}(r)^{2} .
$$

Expanding out the determinant $\sqrt{-g}$ in (5) will give the promised effective mass to $\delta g_{t x}$ and $\delta g_{r x}$. The mass term (5) has the same form as those that arise in the holographic massive gravity theory of [7], albeit with a different radial profile (6).

With these basic explanations of the relevant physics in place, let's now turn to the details. As described above, we focus on the homogenous perturbations to leading order in $\epsilon$. To avoid clutter, we'll set $2 \kappa^{2}=L^{2}=e^{2}=1$ in what follows. It's simplest to keep the phonon as a physical degree of freedom and work in $\delta g_{r x}=0$ gauge. We can describe the perturbations at order $\mathcal{O}\left(\epsilon^{2}\right)$ using the Maxwell, scalar and $r-x$ components of the Einstein equations

$$
\begin{aligned}
\left(f \delta A_{x}{ }^{\prime}\right)^{\prime}+\frac{\omega^{2}}{f} \delta A_{x} & =\frac{\mu}{r_{h}}\left(r^{2} \delta g_{t x}\right)^{\prime} \\
r^{2}\left(\frac{f M^{2}}{r^{2}} \pi^{\prime}\right)^{\prime}+\frac{\omega^{2} M^{2}}{f} \pi & =i \omega \frac{r^{2} M^{2}}{f} \delta g_{t x} \\
\left(r^{2} \delta g_{t x}\right)^{\prime} & =\frac{\mu r^{2}}{r_{h}} \delta A_{x}+\frac{f M^{2}}{i \omega} \pi^{\prime} .
\end{aligned}
$$

The UV boundary condition for the phonon field $\pi$ plays an important role. The fact the we have explicitly, as opposed to spontaneously, broken translational invariance 
means that we require the falloff $\pi(r, t) \sim r^{\Delta_{+}-\Delta_{-}}$at the boundary. In contrast, in situations where translational symmetry is broken spontaneously, the correct boundary condition is that the phonon approaches a constant at the boundary. It is simple to eliminate $\delta g_{t x}$, leaving two coupled equations for $\delta A_{x}$ and $\pi$,

$$
\begin{aligned}
\left(f \delta A_{x}{ }^{\prime}\right)^{\prime}+\frac{\omega^{2}}{f} \delta A_{x} & =\frac{\mu^{2} r^{2}}{r_{h}^{2}} \delta A_{x}+\frac{\mu f M^{2}}{i \omega r_{h}} \pi^{\prime} \\
\frac{1}{r^{2}}\left(\frac{r^{2} f}{M^{2}}\left(\frac{f M^{2}}{r^{2}} \pi^{\prime}\right)^{\prime}\right)^{\prime}+\frac{\omega^{2}}{r^{2}} \pi^{\prime} & =\frac{i \omega \mu}{r_{h}} \delta A_{x}+\frac{f M^{2}}{r^{2}} \pi^{\prime} .
\end{aligned}
$$

The key observation is that these perturbation equations are equivalent to those that arise in massive gravity $[7,9,10]$ with an effective graviton mass $M^{2}(r)$. The phonon mode $\pi$ is related to the extra propagating metric mode $g_{r x}$ in massive gravity through the relation $\pi^{\prime} \rightarrow r^{2} g_{r x}$. Of course this is not a surprise - as we have already emphasized, the two descriptions are gauge equivalent.

Conductivity.-To compute the optical conductivity, we need only solve the perturbation equations subject to the appropriate boundary conditions. Fortunately, many of the relevant calculations have already been performed in the context of massive gravity. It was shown in [10], that the dc conductivity depends only on the behavior of the fields at the infrared horizon. The argument is a generalisation of an earlier observation by Iqbal and Liu [13]. The essence of it goes as follows: the photon $\delta A_{x}$ has an effective mass proportional to the charge density $\mu / r_{h}$; meanwhile, as we described above, the phonon has a mass proportional to $M^{2}$. However, the two modes mix. And it is simple to check that there is a linear combination which is massless and, in the $\omega \rightarrow 0$ limit, does not evolve from the horizon to the boundary. Furthermore, this linear combination carries the information about the conductivity. This means that one can compute the dc conductivity in terms of properties of the horizon of the black hole.

The end result is that the scattering rate is fixed by the effective graviton mass evaluated at the horizon [10],

$$
\Gamma=\frac{s}{4 \pi} \frac{M^{2}\left(r_{h}\right)}{\mathcal{E}+\mathcal{P}},
$$

where the entropy density $s$, energy density $\mathcal{E}$ and pressure $\mathcal{P}$ are thermodynamic functions that are nonzero in the extremal RN black hole background. This result was also obtained for hydrodynamic transport in massive gravity in [9].

The key content of this formula is that the scattering rate is simply determined by the effective graviton mass induced by lattice,

$$
\Gamma \sim M^{2}\left(r_{h}\right) \sim \epsilon^{2} k_{L}^{2} \phi_{0}\left(r_{h}\right)^{2},
$$

where we have dropped the other coefficients on the grounds that they are, to leading order, constants that are independent of temperature.
All that remains is to determine the infrared behavior of the scalar profile $\phi_{0}(r)$ which will govern the temperature dependence of graviton mass (6). But this is straightforward. At $T=0$, the infrared geometry is $\mathrm{AdS}_{2} \times \mathbf{R}^{2}$. As we reviewed in the introduction, this is the holographic manifestation of a locally critical theory. If we denote the radial coordinate in $\mathrm{AdS}_{2}$ as $\zeta$, the regular solution for $\phi$ falls off asymptotically in the infrared as $\phi_{0} \sim \zeta^{\frac{1}{2}-\nu_{k_{L}}}$ where $\nu_{k_{L}}+1 / 2$ is the dimension of the dual operator $\mathcal{O}\left(k_{L}\right)$ in real space. The dependence on the lattice spacing is given by $\nu_{k_{L}}=\sqrt{\frac{1}{4}+\frac{1}{6} m^{2} L^{2}+2 k_{L}^{2} / \mu^{2}}$. Upon taking a Fourier transform, the dimension of the operator in frequency space becomes $\Delta_{k_{L}}=\nu_{k_{L}}-1 / 2$, so we have $\phi_{0} \sim \zeta^{-\Delta_{k_{L}}}$. At finite temperature, the $\mathrm{AdS}_{2}$ geometry terminates in a horizon at $\zeta_{H} \sim T^{-1}$. This means that the effective graviton mass, and hence resistivity, scales as

$$
\rho \sim \epsilon^{2} k_{L}^{2} T^{2 \Delta_{k_{L}}} .
$$

Happily, this is precisely the result of Hartnoll and Hofman [2] that we reviewed in the Introduction.

Closing remarks.-Throughout this Letter, we have relied on the technical crutch of the small-lattice expansion. This allowed us to isolate the phonon mode as the relevant, extra degree of freedom in computing the resistivity. However, we would like to suggest that, even in more complicated situations, the phonon mode continues to dominate the low-temperature physics. Here we offer some suggestions on how this may happen.

Let us first address what would happen if we compute the resistivity to higher order in the lattice strength, $\epsilon$. Further fields-including, most pertinently, the gauge field $A_{t}$-will pick up a spatial modulation and therefore contribute to the effective mass of the graviton at $\mathcal{O}\left(\epsilon^{4}\right)$. The analysis of [2] shows that each such field will give a contribution to the dc conductivity of the form (1). At low temperatures, the charge density $J^{t}\left(2 k_{L}\right)$ is the least irrelevant operator (together with $T_{t t}$, with which it mixes) to get a spatially modulated expectation value and so, although it is sub-leading in the $\epsilon$-expansion, dominates the low-temperature resistivity [2,3].

Although technically more involved, it seems clear how the field theory expectations above are mirrored in the gravity calculation. Clearly, we will have many more perturbed fields in the game. However, among these we expect that there remains a linear combination which is massless and, therefore, does not evolve from the horizon to the boundary. This means that we can focus attention on the far infrared geometry. Here, the gauge field $A_{t}$ is the largest spatially varying field and the fields dominating the perturbation equations are $\delta A_{x}, \delta g_{t x}$, and now the phonon $\delta A_{t}$ arising from the induced ionic lattice. Thus, in the far IR, the perturbation equations reduce to those considered here and resistivity will again be given by (9), but with the 
exponent $\Delta_{k}$ replaced by the appropriate dimension of the ionic lattice (which was computed in [14]).

We note that the conceptual steps sketched above also hold for other situations, such as the ionic lattice, where no simple expansion in the lattice strength is available. Instead, we replace the expansion in $\epsilon$ with an infrared expansion. Of course, this is what one naturally expects for the dc conductivity and, even without an explicit demonstration of the massless mode, it should be possible to extract the leading temperature dependence of the resistivity by a matching calculation $[15,16]$.

Our thanks to Richard Davison, Sean Hartnoll, Sung-Sik Lee, Don Marolf, Koenraad Schalm, and Jan Zaanen for useful conversations. We are grateful to the Newton Institute for hospitality while this project was undertaken. M. B. and D. T. are supported by STFC and by the European Research Council under the European Union's Seventh Framework Programme (FP7/2007-2013), ERC Grant No. STG 279943, "Strongly Coupled Systems."

[1] S. A. Hartnoll, P. K. Kovtun, M. Mueller, and S. Sachdev, Phys. Rev. B 76144502 (2007).
[2] S. A. Hartnoll and D. M. Hofman, Phys. Rev. Lett. 108, 241601 (2012).

[3] G. T. Horowitz, J. E. Santos, and D. Tong, J. High Energy Phys. 07 (2012) 168.

[4] G. T. Horowitz, J. E. Santos, and D. Tong, J. High Energy Phys. 11 (2012) 102.

[5] G. T. Horowitz and J. E. Santos, arXiv:1302.6586.

[6] Y. Ling, C. Niu, J.-P. Wu, and Z.-Y. Xian, J. High Energy Phys. 11 (2013) 006.

[7] D. Vegh, arXiv:1301.0537.

[8] C. de Rham, G. Gabadadze, and A. J. Tolley, Phys. Rev. Lett. 106, 231101 (2011).

[9] R. A. Davison, Phys. Rev. D 88, 086003 (2013).

[10] M. Blake and D. Tong, Phys. Rev. D 88, 106004 (2013).

[11] N. Arkani-Hamed, H. Georgi and M. D. Schwartz, Ann. Phys. (Amsterdam) 305, 96 (2003).

[12] R. A. Battye and J. A. Pearson, Phys. Rev. D 88, 084004 (2013).

[13] N. Iqbal and H. Liu, Phys. Rev. D 79, 025023 (2009).

[14] M. Edalati, J. I. Jottar, and R. G. Leigh, J. High Energy Phys. 10 (2010) 058.

[15] T. Faulkner, H. Liu, J. McGreevy, and D. Vegh, Phys. Rev. D 83, 125002 (2011).

[16] A. Donos and S. A. Hartnoll, Phys. Rev. D 86, 124046 (2012). 